\title{
Impaired sacculocollic reflex in lateral medullary infarction
}

\author{
Seonhye Kim ${ }^{1,2}$, Hyo-Jung Kim ${ }^{2}$ and Ji Soo Kim ${ }^{2 *}$ \\ 1 Department of Neurology, School of Medicine, Pusan National University Yangsan Hospital, Pusan National University, Busan, South Korea \\ 2 Department of Neurology, College of Medicine, Seoul National University Bundang Hospital, Seoul National University, Seoul, South Korea
}

\author{
Edited by: \\ Masahiro lida, Tokai University School \\ of Medicine, Japan \\ Reviewed by: \\ Christoph Helmchen, University of \\ Luebeck, Germany \\ Sergei B. Yakushin, Mount Sinai School \\ of Medicine, USA \\ Americo Migliaccio, Neuroscience \\ Research Australia, Australia \\ ${ }^{*}$ Correspondence: \\ Ji Soo Kim, Department of Neurology, \\ College of Medicine, Seoul National \\ University Bundang Hospital, Seoul \\ National University, 300 Gumi-dong, \\ Bundang-gu, Seongnam-si, \\ Gyeonggi-do, 463-707, Seoul, South \\ Korea.e-mail: jisookim@snu.ac.kr
}

\begin{abstract}
Objective: The aim of this study was to determine saccular dysfunction by measuring cervical vestibular-evoked myogenic potentials (cVEMP) and to correlate abnormality of cVEMP with results of other vestibular function tests in lateral medullary infarction (LMI). Methods: We recorded cVEMP in 21 patients with LMI documented on MRI. cVEMP was induced by a short tone burst and was recorded in contracting sternocleidomastoid muscle while patients turned their heads forcefully to the contralateral side against resistance. Patients also underwent video-oculographic recording of spontaneous, gaze-evoked and head shaking nystagmus (HSN), evaluation of ocular tilt reaction (OTR), measurement of the subjective visual vertical (SVV) tilt, bithermal caloric tests, and audiometry. Results: Nine patients (43\%) showed abnormal cVEMP, unilateral in seven and bilateral in two. The cVEMP abnormalities included decreased p13-n23 amplitude in four, delayed p13/n23 responses in five, and both decreased and delayed responses in two. The abnormal cVEMP was ipsilesional in five, contralesional in two, and bilateral in two. The prevalence of OTR/SW tilt, spontaneous nystagmus, and HSN did not differ between the patients with normal and abnormal cVEMP. Conclusion: cVEMP was abnormal in approximately half of the patients with LMI. The abnormal cVEMP indicates damage to the descending sacculocollic reflex pathway or disruption of commissural modulation between the vestibular nuclei.
\end{abstract}

Keywords: vertigo, vestibular-evoked myogenic potential, Wallenberg syndrome, lateral medullary infarction, saccule

\section{INTRODUCTION}

Cervical vestibular-evoked myogenic potential (cVEMP) refers to inhibitory potentials recorded in the contracting muscles, usually in the sternocleidomastoids (SCM), when loud clicks or short tone burst sounds were applied (Colebatch and Halmagyi, 1992; Colebatch et al., 1994; Wang et al., 1999). cVEMP seems to be generated via a rapidly conducting disynaptic pathway, originating in the saccule and proceeding along the vestibular afferent fibers to the vestibular nuclei, and then through rapidly conducting projections that synapse with SCM muscle (Halmagyi and Colebatch, 1995; Murofushi et al., 1996; Ozeki et al., 1996; Murofushi and Curthoys, 1997). Absence or decreased p13-n23 amplitude, and delayed cVEMP responses indicate the presence of a lesion affecting this pathway (Colebatch and Halmagyi, 1992; Murofushi et al., 2001; Welgampola and Colebatch, 2005).

Several studies have investigated the characteristics and the diagnostic values of cVEMP in central vestibular disorders (Itoh et al., 2001; Chen and Young, 2003; Pollak et al., 2006; Deftereos et al., 2008; Kim and Lee, 2010b; Kim et al., 2010; Tseng and Young, 2010). Due to damage of the vestibular fascicles, vestibular nucleus, or vestibular efferents from the nuclei that are involved in relaying and central processing of peripheral vestibular inputs, lateral medullary infarction (LMI, Wallenberg syndrome) may provide a good anatomical substrate for studying abnormal cVEMP in central vestibulopathies (Baloh et al., 1981; Choi et al., 2007). However, previous studies evaluated cVEMP in only small number of patients and rarely attempted to correlate the findings with the results of other vestibular function tests (Tseng and Young, 2010). We measured
cVEMP in 21 patients with LMI to investigate dysfunction of the saccular pathway, and then correlated the findings with the results of other vestibular function tests.

\section{MATERIALS AND METHODS}

\section{SUBJECTS}

Of the 26 patients who underwent cVEMP with a confirmed diagnosis of isolated LMI without cerebellar lesions at Seoul National University Bundang Hospital from April 2007 to January 2010, 21 patients were included in this study after excluding five patients with a history of vestibular neuritis $(n=1)$ or conductive hearing loss on audiometry ( $n=4$; Tables 1 and 2$)$. The patients included 16 men and the ages ranged from 30 to 76 years (mean $\pm S D=56.4 \pm 12$, median $=60$ ). All the patients received full neurological and neurootological evaluation by the senior author (Ji Soo Kim), and had symptoms and signs consistent with LMI (Tables 1 and 2). Intervals from symptom onset to evaluation of the vestibular function ranged from 1 day to 14 days (median $=3$ day). In particular, 19 of them were evaluated within 1 week of symptom onset.

All experiments followed the tenets of the Declaration of Helsinki. This study was also reviewed and approved by the institutional review board.

\section{NEURO-OTOLOGICAL EXAMINATION}

Spontaneous nystagmus was observed on a video monitor using video Frenzel goggles (SLMED, Seoul, Korea). Patients also underwent recording of eye motion using 3-dimensional videooculography (SMI, Tiltow, Germany; Choi et al., 2007). Detailed 


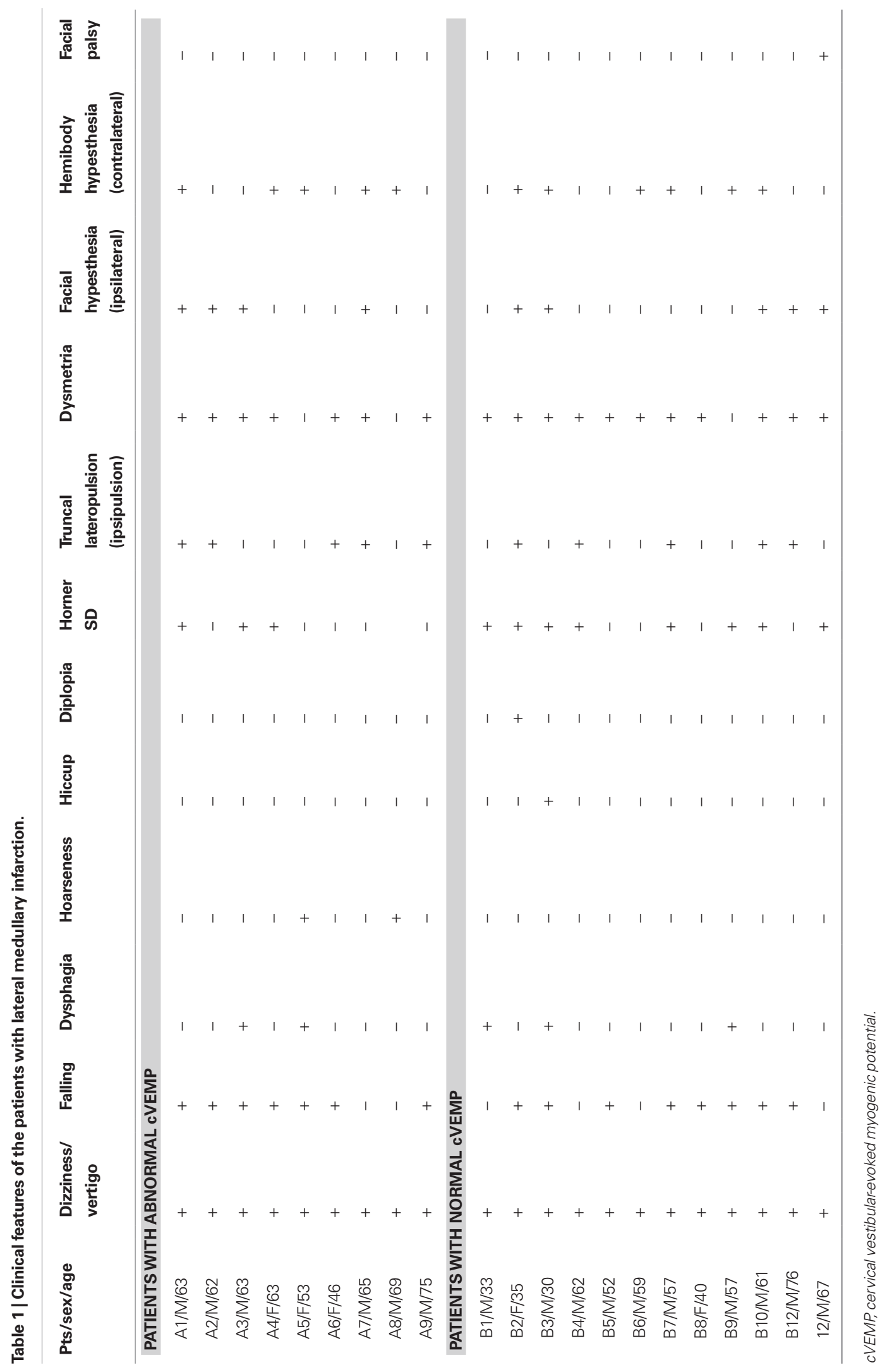




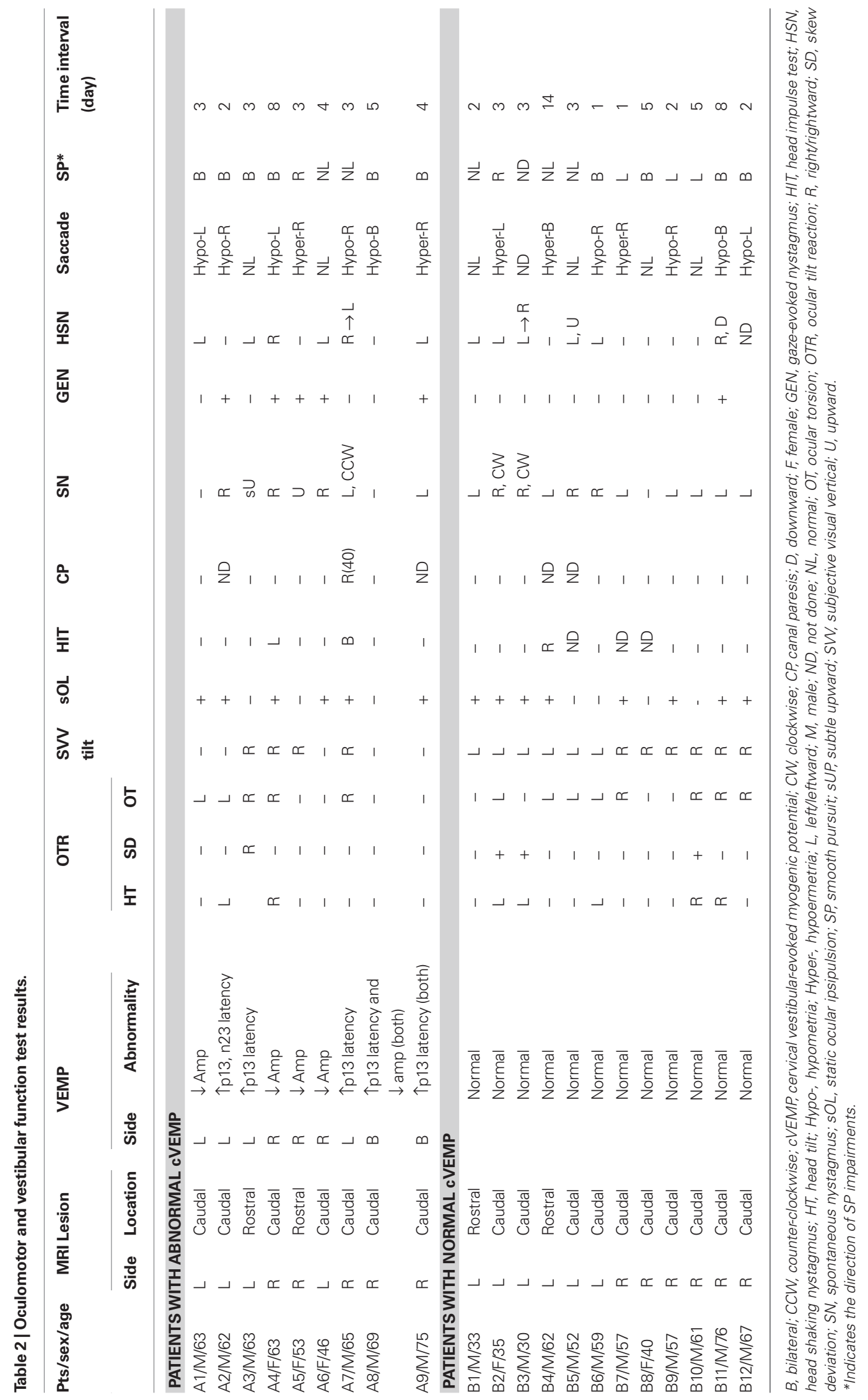


methods of audiometry, fundus photography, measurement of the subjective visual vertical (SVV) tilt, and bithermal caloric tests have been described previously (Choi et al., 2007; Oh et al., 2008).

\section{RECORDING OF cVEMP}

The SCM muscle was activated by actively turning the head contralaterally while lifting the head in the supine position. Patients had to hold this position precisely to achieve a constant tonic activation of the SCM during the whole recording periods. Electromyographic activities of the SCM were recorded using surface electrodes from the upper half of the SCM muscle with a ground electrode on the forehead and a reference electrode over the upper sternum. cVEMP was detected using a Nicolet Viking Select unit (Nicolet Biomedical, Madison, WI, USA). A short alternating tone bursts ( $110 \mathrm{~dB} \mathrm{nHL}$; $500 \mathrm{~Hz}$; ramp = $2 \mathrm{~ms}$; plateau $=3 \mathrm{~ms}$ ) were given at $2.1 \mathrm{~Hz}$ monaurally using a headphone. The analysis time for each stimulus was $50 \mathrm{~ms}$ and responses for up to 200 stimuli were averaged for each test. The signal was filtered at a bandpass of $30-1500 \mathrm{~Hz}$, and the mean values of at least two trials were obtained from each ear.

During the recording, the amplified electromyographic activities of SCM were also monitored and digitized at $1 \mathrm{KHz}$ using an $\mathrm{AD}$ converter (NI PCI-4461, National Instruments, Austin, TX, USA). Using Labview (National Instruments, Austin, TX, USA) program, the peak-to-peak amplitudes were analyzed and mean tonic activation during the recording was calculated. The absolute cVEMP amplitude was then normalized against mean tonic activation of SCM during the recording.

To compare the normalized p13-n23 amplitude of cVEMP responses in the patients, the interaural difference ratio of the normalized amplitude $\left(\operatorname{IAD}_{\text {amp }}, \%\right)$ was also calculated using $\left[\left(\left|A_{\mathrm{R}}-A_{\mathrm{L}}\right|\right) /\right.$ $\left.\left(A_{\mathrm{R}}+\mathrm{A}_{\mathrm{L}}\right) \times 100\right]$, where $A_{\mathrm{R}}$ is the normalized p13-n23 amplitude on the right side and $A_{\mathrm{L}}$ is the amplitude on the left side (Welgampola and Colebatch, 2005). In patients, decreased cVEMP amplitude was determined when the normalized p13-n23 amplitude was less than mean $-2 \mathrm{SD}$ or $\mathrm{IAD}_{\text {amp }}$ was larger than mean $+2 \mathrm{SD}$ of normal controls. Regarding $\mathrm{p} 13$ and $\mathrm{n} 23$ latencies, absolute values and interaural differences were obtained to determine delayed responses when $\mathrm{p} 13 /$ n23 latencies or interaural latency differences (IAD) were greater than mean $+2 \mathrm{SD}$ of normal controls.

The cutoff values of abnormal cVEMP data are 20.6\% $($ mean $+2 \mathrm{SD})$ for $\mathrm{IAD}_{\text {amp }}, 16.5 \mathrm{~ms}($ mean $+2 \mathrm{SD})$ for p13 patency, and $27.3 \mathrm{~ms}$ (mean $+2 \mathrm{SD}$ ) for $\mathrm{n} 23$ latency. Detailed methods and normative data of cVEMP have been reported previously (Kim et al., 2010; Shin et al., 2009).

\section{BRAIN IMAGING}

MRI was performed with a 1.5-T unit (Intera, Philips Medical Systems, Best, the Netherlands) using our standard imaging protocol (axial turbo spin-echo T2-wighted imaging, axial spin-echo T1-weighted imaging, and axial gradient-echo imaging; Choi et al., 2007). Diffusion-weighted imaging (DWI) was additionally performed in 10 patients using the following parameters; $b=1000,4119 / 89$ (repetition time/echo time), a section thickness of $5 \mathrm{~mm}$, a matrix of $128 \times 128$ (interpolated to $256 \times 256$ ), and a field-of-view of $220 \mathrm{~mm}$. All the patients underwent MRIs from $1 \mathrm{~h}$ to 14 days (mean $\pm \mathrm{SD}=1.7 \pm 3.0$ days, median $=1$ day) of symptom onset.

\section{LESION ANALYSES}

The medulla scanned in the axial plane was divided into the rostral, middle, and caudal portions by an approximately equal division along its long axis from the pontomedullary junction superiorly to the level of the foramen magnum inferiorly (Choi et al., 2007). The rostral medulla was characterized by massive bulging of the dorso-lateral area due to the restiform body, the middle medulla by bulging of the lateral surface due to the inferior olive, and the caudal medulla by a relatively round shape without bulging of the lateral surface (Kim et al., 1994). According to the location of the lesions observed on MRI, patients were then classified as either the rostral or caudal group; the "rostral group" included the patients with MRI lesions in the rostral medulla or both in the rostral and middle medulla while the "caudal group" comprised the patients with lesions in the caudal medulla or both in the middle and caudal medulla (Kim, 2003). Two neurologists, who were blinded to the clinical information, determined the rostrocaudal extent of the lesions and involvement of the vestibular nuclei on the diffusion-weighted and T2-weighted axial images using the diagram previously reported (Kim, 2003).

To determine which brainstem structures were responsible for abnormal cVEMP, we compared the MRI lesions between the patients with normal and abnormal cVEMP. Using MRIcro software ${ }^{1}$, DWI or T2-weighted MRI lesions of each patient were mapped onto slices of a T1-weighted template MRI scan from the Montreal Neurological Institute $(\mathrm{MNI})^{2}$. This template is approximately oriented to match Talairach space (Talairach and Tournous, 1988). To facilitate the lesion analysis, we flipped the regions of interest (ROIs) of the patients with left-sided lesion, and finally arranged all the ROIs on the right side. Lesions were mapped onto the slices that correspond to $z$-coordinates $-56,-52,-48$, and $-44 \mathrm{~mm}$ in Talairach coordinates.

In each group of patients, we obtained overlap images using lesion density plots. Then, subtraction of the overlapped lesions in normal cVEMP group from those in abnormal group yielded another percentage overlay plot which indicated relative incidence of damage specific for abnormal cVEMP.

We also determined any difference in the distribution of MRI lesions between the groups by comparing prevalence of the lesions involving the vestibular nuclei. To avoid information bias, all MRI lesions were checked and mapped by a neurologist unaware of clinical information.

\section{STATISTICAL ANALYSES}

Fisher's exact tests were used to compare the prevalence of spontaneous nystagmus, gaze-evoked nystagmus (GEN), head shaking nystagmus (HSN), and ocular tilt reaction (OTR)/abnormal SVV tilt between the normal and abnormal cVEMP groups. Non-parametric correlations ( $\chi^{2}$-test) were used to compare the horizontal direction (ipsilesional versus contralesional) of spontaneous nystagmus, HSN and the direction of OTR/abnormal SVV tilt between the groups. Also the rostrocaudal extent of the lesions and involvement of the vestibular nuclei were compared

${ }^{1}$ www.mricro.com

${ }^{2}$ www.bic.mni.mcgill.ca/cgi/icbm_view 
using non-parametric correlations $\left(\chi^{2}\right.$-test $)$ between the groups. All tests were performed using SPSS (version 12.0; SPSS, Chicago, IL, USA) and $p<0.05$ were considered significant.

\section{RESULTS}

\section{CLINICAL CHARACTERISTICS}

All patients suffered from vertigo/dizziness, and 15 patients fell to the side of MRI lesions on standing. Other findings included ipsilesional Horner syndrome $(n=11)$, hypesthesia in the contralesional extremities $(n=11)$, ipsilesional facial hypesthesia $(n=9)$, dysphagia $(n=5)$, hoarseness $(n=2)$, diplopia $(n=1)$, and ipsilesional facial palsy $(n=1$; Table 1$)$. However, the prevalence of these findings did not differ between the normal and abnormal cVEMP groups $(p>0.05)$.

The data on spontaneous nystagmus and HSN will be described in the following section on other vestibular findings and Table 2.

\section{ABNORMALITIES OF cVEMP}

Abnormal cVEMP responses were recorded in nine (43\%) patients, unilateral in seven and bilateral in two (Figures 1A, 2). Overall, 11 ears showed abnormal cVEMP responses which included decreased p13-n23 amplitude in six, delayed p13/n23 responses in six, and both decreased and delayed responses in two ears (Table 2). Of the seven patients with unilateral cVEMP abnormalities, five showed abnormal cVEMP in the imaged side of the lesion (Figures 1A1,2A) and two had abnormal cVEMP in the contralateral side (Figures 1A2, 2B; Table 2). No difference was found between the patients with normal and abnormal cVEMP in terms of age, sex, and interval from symptom onset to cVEMP examination ( $p>0.05$; Table 3$)$.

\section{IMAGING FINDINGS}

Eleven patients with isolated LMI had right sided lesions while 10 patients showed lesions in the left side. The MRI lesions were found in the rostral medulla in 4, in the middle medulla in 14, and in the caudal medulla in 17 patients. Overall, 4 patients were classified into the rostral group and 17 patients were classified into the caudal group. The rostrocaudal extent of the lesions did not differ between the abnormal and normal cVEMP groups ( $p=0.586$; Table 2$)$. The lesion involving the vestibular nuclei was found in 14 patients, 6 $(67 \%)$ in abnormal and $8(67 \%)$ in normal cVEMP group. The proportion of patients with lesions involving the area of the vestibular nuclei did not differ between the groups, either $(p=0.681)$. The lesion overlay plots (Figures A1A,B in Appendix on the Frontiers in Neuro-otology $y^{\otimes 3}$ ) and a subtraction image (Figure A1C in Appendix on the Frontiers in Neuro-otology $y^{\oplus 4}$ ) did not reveal any overlapped structure responsible for abnormal cVEMP in the medulla.

\section{OTHER VESTIBULAR FINDINGS}

Eighteen $(85.7 \%)$ patients showed spontaneous nystagmus that was horizontal in 13, mixed horizontal-torsional in three, and upbeat in two (Table 2). The direction of the horizontal nystagmus was ipsilesional in $3(3 / 16,19 \%)$ and contralesional in $13(13 / 16,81 \%)$ patients. GEN was observed in $6(29 \%)$ patients.

\footnotetext{
${ }^{3}$ http://frontiersin.org/neuro-otology
} ${ }^{4}$ http://frontiersin.org/neuro-otology
Head shaking nystagmus was observed in $12(60 \%)$ of 20 patients who had the test. The horizontal direction of HSN was mostly ipsilesional $(11 / 12,92 \%)$ while it was contralesional only in one patient (Table 2). In 7 of 13 patients with contralesional spontaneous nystagmus, HSN beat in the opposite direction of the spontaneous nystagmus. In contrast, one patient (A9) with contralesional spontaneous nystagmus showed an augmentation of the nystagmus after horizontal head shaking.

Fourteen (67\%) patients showed at least one component of OTR. The direction of OTR was all ipsiversive except one (patient A3) who showed right skew deviation and clockwise ocular torsion in the right eye from left-sided lesion. Sixteen (76\%) patients showed SVV tilt that was ipsiversive in 15 (94\%) and contraversive only in one patient (patient A3) with contraversive ocular torsion.

Canal paresis was detected only in one (patient A7) of the 17 patients who had the bithermal caloric tests (Table $\mathbf{1}$ ).

\section{COMPARISON OF cVEMP ABNORMALITY WITH OTHER VESTIBULAR FINDINGS}

Between the abnormal and normal cVEMP groups, no differences were found in the prevalence of spontaneous nystagmus, HSN, and OTR/SVV tilt ( $p>0.05$, Table 3$)$. Only the proportion of GEN

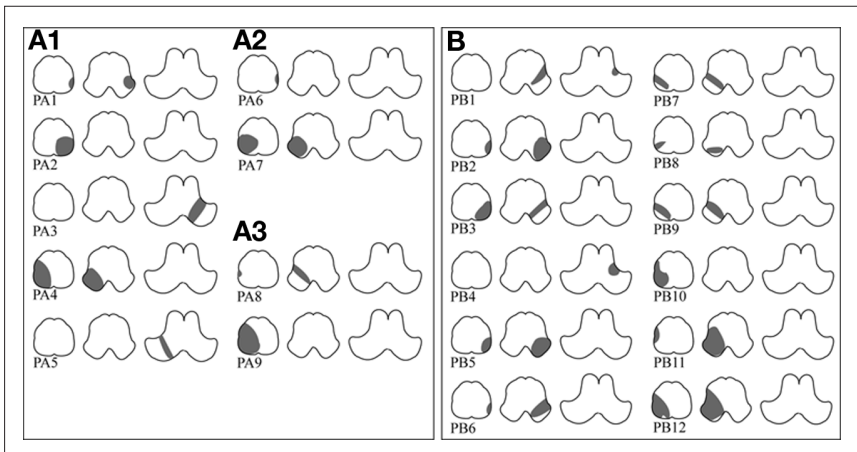

FIGURE 1 |Topographic distribution of lateral medullary infarction in patients with abnormal (A) and normal (B) cervical vestibular-evoked myogenic potential (cVEMP). In the abnormal group (A), the lesions are also arranged according to the side of cVEMP abnormality; ipsilesional (A1), contralesional (A2), and bilateral (A3). The lesions in normal group are shown in (B).

Table 3 | Comparison of demographics and associated findings between the groups.

\begin{tabular}{llll}
\hline & $\begin{array}{l}\text { Patients with abnormal } \\
\text { cVEMP }(\boldsymbol{n}=\mathbf{9})\end{array}$ & $\begin{array}{l}\text { Patients with normal } \\
\text { cVEMP ( } \boldsymbol{n = 1 2 )}\end{array}$ & p-value \\
\hline Age & $62.1 \pm 8.4$ & $52.4 \pm 14.6$ & 0.059 \\
Sex (M:F) & $6: 3$ & $10: 2$ & 0.611 \\
Interval (days) & 4.1 & 4 & 0.982 \\
SN & 5 & 11 & 0.705 \\
GEN & 5 & 1 & 0.046 \\
HSN & 6 & 7 & 0.813 \\
OTR/SW tilt & 7 & 12 & 0.171
\end{tabular}

CVEMP, cervical vestibular evoked myogenic potential; SN, spontaneous nystagmus; GEN, gaze-evoked nystagmus; HSN, head shaking nystagmus; OTR, ocular tilt reaction; SVV, subjective visual vertical. 

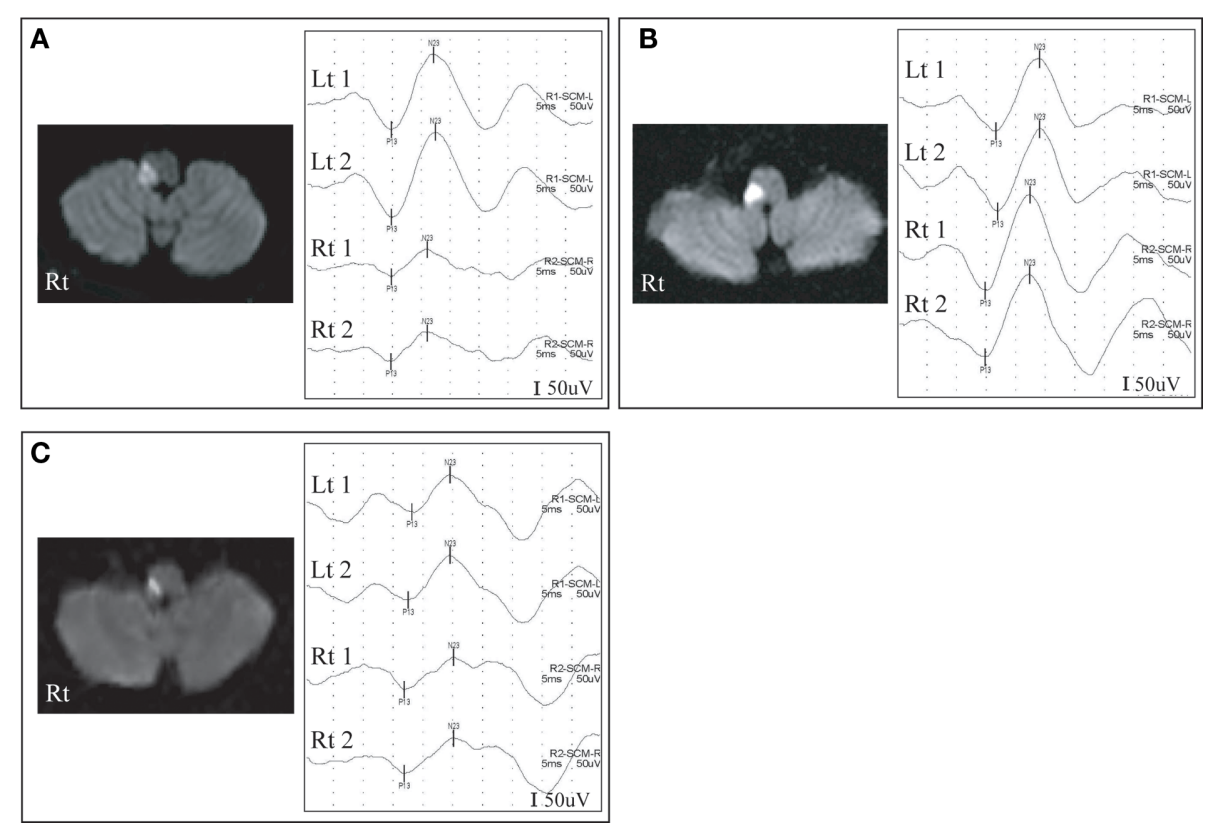

FIGURE 2 |Abnormal cervical vestibular-evoked myogenic potentials (cVEMP) and diffusion-weighted magnetic resonance images (DWI). A patient (patient 4) with right lateral medullary infarction (LMI) shows decreased amplitude on cVEMP in the ipsilesional side (A) while another patient (patient 7) with right LMI exhibits delayed p13 responses in the contralesional side (B). The other patient (patient 8) with right LMI shows delayed p13 responses and decreased amplitude in the ipsi- and contralesional side (C). was higher in the abnormal $(5 / 9,56 \%)$ than the normal cVEMP group $(1 / 12,8 \%)$ with a marginal statistical significance $(p=0.046$, Table 3 ).

\section{DISCUSSION}

In this study, nine $(43 \%)$ of the 21 patients with LMI showed abnormal VEMP responses while only one patient exhibited canal paresis. In contrast to the caloric testing that evaluates the VOR originating from the horizontal semicircular canal, cVEMP assesses the sacculocollic reflex which travels downward in the lower brainstem (Wilson et al., 1995; Uchino et al., 1997; Kushiro et al., 1999; Chen and Young, 2003). LMI may affect cVEMP by damaging the vestibular fascicles, the vestibular nuclei, or descending fibers from the vestibular nuclei to the neck muscles. However, normal calorics in almost all of our patients preclude the vestibular fascicles as a lesion site responsible for abnormal cVEMP (Francis et al., 1992).

In primates, saccular projections reach the superior, lateral, and inferior vestibular nuclei (Imagawa et al., 1998; Newlands et al., 2003), and proceed bilaterally to the spinal cord (Uchino et al., 1997). However, sound-evoked cVEMPs recorded from the neck are almost completely unilateral (Colebatch et al., 1994; Murofushi et al., 1996; Welgampola and Colebatch, 2005). The sacculocollic inhibitory signal may travel down from the inferior vestibular nucleus mostly along the medial VST to the ipsilateral SCM muscle (Colebatch et al., 1994; Robertson and Ireland, 1995; Wilson et al., 1995; Murofushi et al., 1996; Kim et al., 2010). Previously, patients with lower brainstem infarction including LMI showed abnormal cVEMP responses in the lesion side (Itoh et al., 2001; Pollak et al., 2006; Kim and Lee, 2010b; Kim et al., 2010; Tseng and
Young, 2010). However, some of our patients (four out of nine with abnormal cVEMP) showed abnormal cVEMP in the contralesional side. These findings suggest an existence of another pathway in the lower brainstem that may affect the contralateral sacculocollic pathway. Likewise the central vestibular pathways mediating the inputs from the horizontal semicircular canal, the otolithic system receives inputs from the contralateral primary afferents via the commissural fibers. While the commissural fibers inhibit the contralateral second-order neurons in the horizontal canal pathways (Shimazu and Precht, 1966), approximately $16 \%$ of the second-order neurons activated by the saccule receive polysynaptic commissural facilitation and $10 \%$ had commissural inhibition (Uchino et al., 2001). Thus, the contralesional or bilateral abnormalities of cVEMP in some of our patients with LMI indicates isolated or combined damage to the commissural fibers mediating contralateral modulation of the saccular input. In this study, the prevalence of OTR/abnormal SVV tilt was higher than that of abnormal cVEMP responses. According to experimental studies adopting single-cell reconstructions, utricular afferents terminate mainly in the inferior vestibular nucleus while the fibers originating from the saccule terminate mainly in the lateral and inferior vestibular nuclei, and sometimes in the superior vestibular nucleus (Imagawa et al., 1995; Sato et al., 1996). While only $10 \%$ of commissural fibers inhibit the contralateral second-order neurons in the saccular pathways, more than half of the secondorder neurons and approximately half of the third-order neurons activated by the utricle receive commissural inhibition from the contralateral utricular nerve (Uchino et al., 2001). The occasional dissociation between cVEMP abnormalities and OTR/SVV tilt suggests differential involvements of the otolithic pathways in the 
central vestibular structures. Otherwise, the OTR/SVV tilt may have resulted from damage to the vertical semicircular pathways (Brandt and Dieterich, 1994).

In the present study, the rostrocaudal distribution of the MRI lesions did not correlate with the cVEMP abnormalities. Previous reports on cVEMP in five patients with LMI showed abnormalities only in those with caudal lesions (Tseng and Young, 2010). However, other studies also reported abnormal cVEMP responses in lesions involving the rostral medulla or pontomedullary junction (Itoh et al., 2001; Pollak et al., 2006; Kim et al., 2010). Primary afferents from the saccule terminate mainly in the ipsilateral lateral vestibular nucleus, but are also connected to the inferior and superior vestibular nuclei. Furthermore, there are interconnections among the vestibular nuclei in both sides (Uchino et al., 2001), which may explain the abnormal cVEMP in our patients with upper medullary lesions.

Our patients with abnormal cVEMP showed decreased or delayed responses of cVEMP, and delayed response in the presence of normal amplitude was observed in four. Decreased or no responses of cVEMP are typical of ischemic damage to the sacculocollic reflex pathways, and delayed responses are frequently reported in the retrolabyrinthine demyelinating disorders. However, the delayed responses with normal amplitude were also reported in ischemic lesions (Kim et al., 2010), and may indicate an incomplete damage leading to demyelination.

In our patients, the horizontal direction of HSN was mostly ipsilesional and even the patients with contralesional spontaneous nystagmus showed ipsilesional HSN. These results are consistent with our previous findings in Wallenberg syndrome (Choi et al., 2007). The mostly ipsilesional HSN in LMI may be due to unilaterally impaired uvulonodular inhibition over the velocity storage (Choi et al., 2007).

\section{REFERENCES}

Baloh, R. W., Yee, R. D., and Honrubia, V. (1981). Eye movements in patients with Wallenberg's syndrome. Ann. N. Y. Acad. Sci. 374, 600-613.

Brandt, T., and Dieterich, M. (1994). Vestibular syndromes in the roll plane: topographic diagnosis from brainstem to cortex. Ann. Neurol. 36, 337-347.

Chen, C. H., and Young, Y. H. (2003). Vestibular evoked myogenic potentials in brainstem stroke. Laryngoscope 113, 990-993.

Choi, K. D., Oh, S. Y., Park, S. H., Kim, J. H., Koo, J. W., and Kim, J. S. (2007). Headshaking nystagmus in lateral medullary infarction: patterns and possible mechanisms. Neurology 68, 1337-1344.

Colebatch, J. G., and Halmagyi, G. M. (1992). Vestibular evoked potentials in human neck muscles before and after unilateral vestibular deafferentation. Neurology 42, 1635-1636.

Colebatch, J. G., Halmagyi, G. M., and Skuse, N. F. (1994). Myogenic potentials generated by a click-evoked vestibulocollic reflex. J. Neurol. Neurosurg. Psychiatr. 57, 190-197.
Deftereos, S. N., Panagopoulos, G., Eleftheriadou, A., Korres, S., Georgonikou, D., Kandiloros, D., and Karageorgious, C. E. (2008). Using vestibular evoked myogenic potentials to localise brainstem lesions. A preliminary report. $B-E N T$ 4, 215-219.

Francis, D. A., Bronstein, A. M., Rudge, P., and du Boulay, E. P. (1992). The site of brainstem lesions causing semicircular canal paresis: an MRI study. J. Neurol. Neurosurg. Psychiatr. 55, 446-449.

Halmagyi, G. M., and Colebatch, J. G. (1995). Vestibular evoked myogenic potentials in the sternomastoid muscle are not of lateral canal origin. Acta Otolaryngol. Suppl. 520(Pt 1), 1-3.

Imagawa, M., Graf, W., Sato, H., Suwa, H., Isu, N., Izumi, R., and Uchino, Y. (1998). Morphology of single afferents of the saccular macula in cats. Neurosci. Lett. 240, 127-130.

Imagawa, M., Isu, N., Sasaki, M., Endo, K., Ikegami, H., and Uchino, Y. (1995). Axonal projections of utricular afferents to the vestibular nuclei and the

Positive head impulse test is typical of the peripheral vestibular disorders, but may be observed in lesions involving the central vestibular structures, especially the vestibular nucleus (Kim and Lee, 2010a). In this study, bedside head impulse test showed catch-up saccades in three patients during head rotations to the contralesional side or in both horizontal directions. Damage to the vestibular nuclei or the commissural fibers might explain the abnormal head impulse test, but the mechanisms require further exploration using a quantitative evaluation.

Our study failed to reveal any overlapped structure responsible for abnormal cVEMP in LMI. This may be ascribed to poor spatial resolution of the current MRI techniques, especially for DWI. Even though DWI is highly sensitive in detecting acute brainstem lesions, even when T2-weighted and fluid-attenuated inversion recovery MRIs are normal, and is effective in differentiating acute infarctions from chronic ones (Lutsep et al., 1997), DWI may fail to reveal ischemic lesions during the hyperacute phase (Wang et al., 1999) and the lesion extent on DWI may be smaller than the area with functional impairments. For example, one of our patients (A6) with abnormal cVEMP showed a restricted lesion in the laterocaudal medulla. However, the associated spontaneous nystagmus, GEN, and HSN suggest a more widespread functional impairment than the MRI lesion. Otherwise, the sacculocollic reflex pathways may be more variable in their anatomical connections through the vestibular nuclei (Uchino et al., 2001).

\section{ACKNOWLEDGMENTS}

This study was supported by grant of the Korea Health 21 R\&D Project, Ministry of Health and Welfare, Republic of Korea (A080750). The authors thank Jong-Hee Lee for experimental assistance.

abducens nucleus in cats. Neurosci. Lett. 186, 87-90.

Itoh, A., Kim, Y. S., Yoshioka, K., Kanaya, M., Enomoto, H., Hiraiwa, F., and Mizuno, M. (2001). Clinical study of vestibular-evoked myogenic potentials and auditory brainstem responses in patients with brainstem lesions. Acta Otolaryngol. Suppl. 545, 116-119.

Kim, H. A., and Lee, H. (2010a). Isolated vestibular nucleus infarction mimicking acute peripheral vestibulopathy. Stroke 41, 1558-1560.

Kim,J.S., and Lee, H. (2010b). "Vertigo and imbalance: Clinical neurophysiology of the vestibular system," in Handbook of Clinical Neurophysiology, eds S.D.Z. Eggers and D.S. Zee (Amsterdam: Elsevier), 451-477.

Kim, J. S. (2003). Pure lateral medullary infarction: clinical-radiological correlation of 130 acute, consecutive patients. Brain 126, 1864-1872.

Kim, J.S., Lee, J. H., Suh, D. C., and Lee, M. C. (1994). Spectrum of lateral medullary syndrome. Correlation between clinical findings and magnetic resonance imaging in 33 subjects. Stroke 25, 1405-1410.

Kim, S., Lee, H. S., and Kim, J. S. (2010). Medial vestibulospinal tract lesions impaired saccule-collic reflexes. $J$. Neurol. 257, 825-832.

Kushiro, K., Zakir, M., Ogawa, Y., Sato, H., and Uchino, Y. (1999). Saccular and utricular inputs to sternocleidomastoid motoneurons of decerebrate cats. Exp. Brain Res. 126, 410-416.

Lutsep, H. L., Albers, G. W., DeCrespigny, A., Kamat, G. N., Marks, M. P., and Moseley, M. E. (1997). Clinical utility of diffusion-weighted magnetic resonance imaging in the assessment of ischemic stroke. Ann. Neurol. 41, 574-580.

Murofushi, T., and Curthoys, I. S. (1997). Physiological and anatomical study of click-sensitive primary vestibular afferents in the guinea pig. Acta Otolaryngol. 117, 66-72.

Murofushi, T., Halmagyi, G. M., Yavor, R. A., and Colebatch, J. G. (1996). Absent vestibular evoked myogenic potentials in vestibular neurolabyrinthitis. An indicator of inferior vestibular nerve 
involvement? Arch. Otolaryngol. Head Neck Surg. 122, 845-848.

Murofushi, T., Shimizu, K., Takegoshi, H., and Cheng, P. W. (2001). Diagnostic value of prolonged latencies in the vestibular evoked myogenic potential. Arch. Otolaryngol. Head Neck Surg. 127, 1069-1072.

Newlands, S. D., Vrabec, J.T., Purcell, I. M., Stewart, C.M.,Zimmerman, B.E., and Perachio, A. A. (2003). Central projections of the saccular and utricular nerves in macaques. J. Comp. Neurol. 466, 31-47.

Oh, Y.M., Oh, D. H., Jeong, S. H., Koo, J.W., and Kim, J.S. (2008). Sequential bilateral hearing loss in multiple sclerosis. Ann. Otol. Rhinol. Laryngol. 117, 186-191.

Ozeki, H., Matsuzaki, M., and Murofushi, T. (1996). Vestibular evoked myogenic potentials in patients with bilateral profound hearing loss. ORL J. Otorhinolaryngol. Relat. Spec. 61, 80-83.

Pollak, L., Kushnir, M., and Stryjer, R. (2006). Diagnostic value of vestibular evoked myogenic potentials in cerebellar and lower-brainstem strokes. Neurophysiol. Clin. 36, 227-233.

Robertson, D. D., and Ireland, D. J. (1995). Vestibular evoked myogenic potentials. J. Otolaryngol. 24, 3-8.

Sato, H., Endo, K., Ikegami, H., Imagawa, M., Sasaki, M., and Uchino, Y. (1996). Properties of utricular nerve-activated vestibulospinal neurons in cats. Exp. Brain Res. 112, 197-202.

Shimazu, H., and Precht, W. (1966). Inhibition of central vestibular neurons from the contralateral labyrinth and its mediating pathway. J. Neurophysiol. 29, 467-492.

Shin, J. Y., Song, H. S., Koo, J. W., Lee, H. S., and Kim, J.S. (2009). Abnormal vestibular evoked myogenic potentials in medial medullary infarction. J. Clin. Neurol. 5, 101-103.

Talairach, J., and Tournous, P. (1988). Co-planar Stereotaxic Atlas of the Human Brain: 3-Dimensional Proportional System - An Approach to Cerebral Imaging. New York: Thieme. Tseng, C. L., and Young, Y. H. (2010). Topographical correlations of lateral medullary infarction with caloric- and vestibular-evoked myogenic potential results. Eur. Arch. Otorhinolaryngol. 267, 191-195.

Uchino, Y., Sato, H., Sasaki, M., Imagawa, M., Ikegami, H., Isu, N., and Graf, W. (1997). Sacculocollic reflex arcs in cats. J. Neurophysiol. 77, 3003-3012.

Uchino, Y., Sato, H., Zakir, M., Kushiro, K., Imagawa, M., Ogawa, Y., Ono, S., Meng, H., Zhang, X., Katsuta, M., Isu, N., and Wilson, V. J. (2001). Commissural effects in the otolith system. Exp. Brain Res. 136, 421-430.

Wang, P.Y., Barker, P. B., Wityk, R. J., Ulug, A. M., van Zijl, P. C., and Beauchamp, N. J. Jr. (1999). Diffusion-negative stroke: a report of two cases. AJNR Am. J. Neuroradiol. 20, 1876-1880.

Welgampola, M. S., and Colebatch, J. G. (2005). Characteristics and clinical applications of vestibular-evoked myogenic potentials. Neurology 64, 1682-1688.

Wilson, V. J., Boyle, R., Fukushima, K., Rose, P. K., Shinoda, Y., Sugiuchi, Y., and Uchino, Y. (1995). The vestibulocollic reflex. J. Vestib. Res. 5, 147-170.

Conflict of Interest Statement: The authors declare that the research was conducted in the absence of any commercial or financial relationships that could be construed as a potential conflict of interest.

Received: 15 September 2010; accepted: 03 February 2011; published online: 15 February 2011.

Citation: Kim S, Kim H-J and Kim JS (2011) Impaired sacculocollic reflex in lateral medullary infarction. Front. Neur. 2:8. doi: 10.3389/fneur.2011.00008

This article was submitted to Frontiers in Neuro-otology, a specialty of Frontiers in Neurology.

Copyright $\odot 2011 \mathrm{Kim}$, Kim and Kim. This is an open-access article subject to an exclusive license agreement between the authors and Frontiers Media SA, which permits unrestricted use, distribution, and reproduction in any medium, provided the original authors and source are credited. 

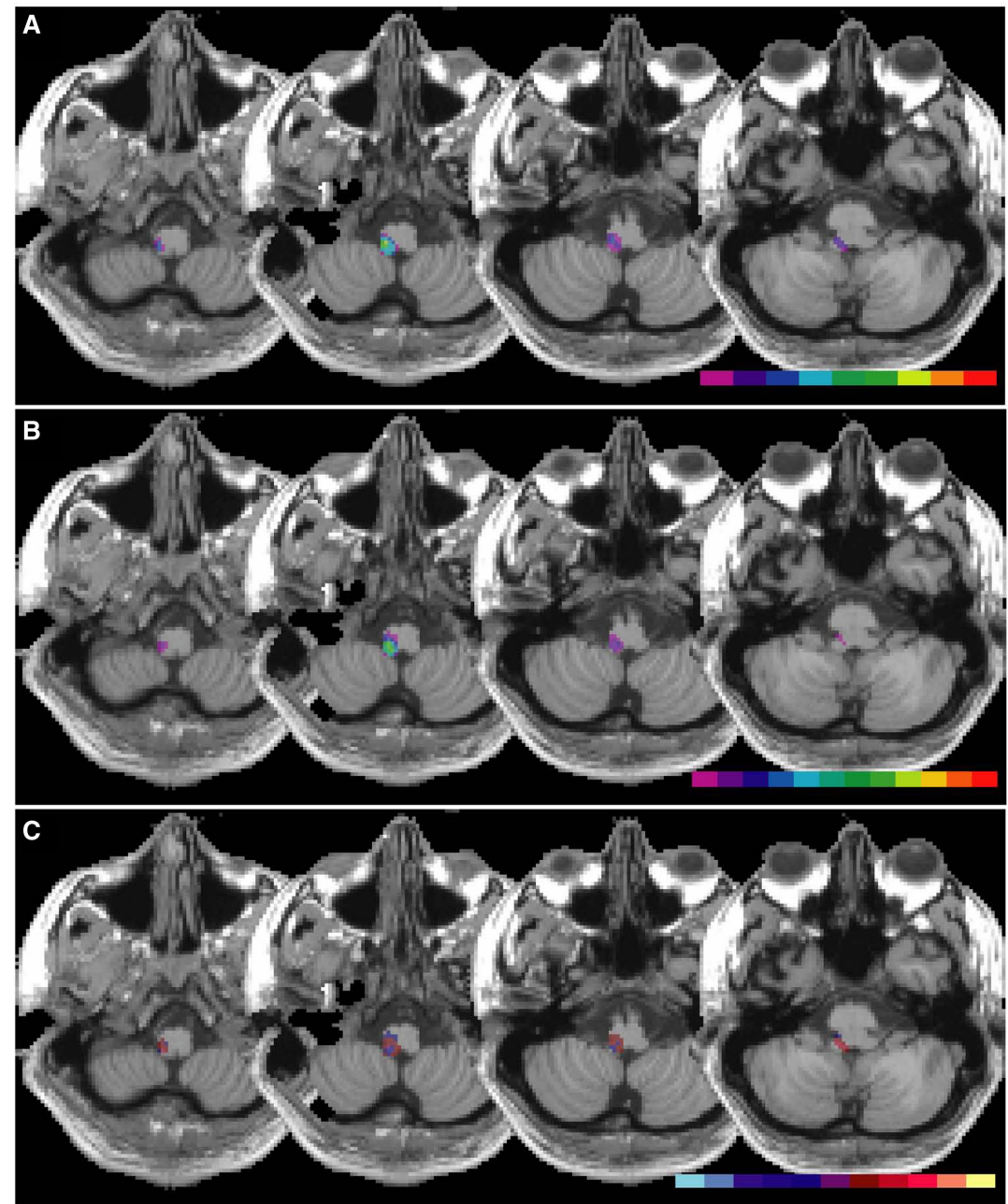

FIGURE A1 | Lesion overlay plots and subtraction images. (A) Lesion overlay plot in the patients with abnormal cervical vestibular-evoked myogenic potentials (cVEMP; $n=9$ ). The number of overlapping lesions is illustrated by different color coding with increasing frequencies from violet $(n=1)$ to red $(n=9)$. (B) Lesion overlay plot in the patients with normal cVEMP $(n=12)$. The number of overlapping lesions is illustrated by different color coding with increasing frequencies from violet $(n=1)$ to red $(n=12)$. (C) In this figure, the overlapped lesions in patients with normal cVEMP were subtracted from those of patients with abnormal cVEMP. The percentages of overlapping lesions after subtraction are indicated by five different color codings with increasing frequencies from dark red (difference $1-20 \%$ ) to white-yellow (difference $80-100 \%$ ). The colors from dark blue (difference -1 to $-20 \%$ ) to light blue (difference -81 to $-100 \%$ ) indicate the regions damaged more frequently in the patients with normal cVEMP than in those with abnormal cVEMP.Talairach $z$-coordinates of each transverse slice are given. 